\title{
Evolutionary sets of safe ship trajectories with speed reduction manoeuvres within traffic separation schemes
}

\author{
Rafał Szłapczyński, Ph. D. \\ Gdańsk University of Technology
}

\begin{abstract}

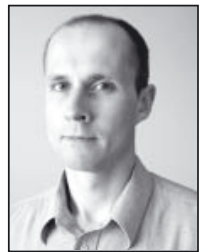

In the previous paper the author presented the evolutionary ship trajectory planning method designed to support Traffic Separation Schemes (TSS). This time the extensions of this method are described which allow to combine evolutionary trajectory planning with speed reduction manoeuvres. On TSS regions with higher than usual density of traffic and smaller distances between ships, the course alterations alone are not always sufficient or effective means of collision avoidance. Therefore they must be supplemented by speed reduction manoeuvres to a larger extent than on open waters. The paper includes a brief description of the optimisation problem, descriptions of the new elements of the method (fitness function, algorithms and the evolutionary cycle) and the examples of how the extended method successfully solves the problems unsolvable without applying speed reduction.
\end{abstract}

Key words: collision avoidance manoeuvres; track planning; Traffic Separation Schemes; speed reduction

\section{INTRODUCTION}

In an earlier paper [10] the author has presented the Evolutionary Sets of Safe Ship Trajectories (ESoSST) method working for Traffic Separation Schemes (TSS) and possible to be applied in Vessel Traffic Service (VTS) centres. This method combined evolutionary approach [9] with goals typical for methods based on games theory [7]. However, unlike other methods utilizing evolutionary algorithms $[9,16]$, genetic algorithms [2, 6, 13] and related heuristics [8, 12, 14, 15], instead of finding an optimal single ship trajectory only, the author's method searches for an optimal set of safe trajectories of all ships involved in an encounter. In the present paper the extensions of this method are described which allow to combine evolutionary trajectory planning with speed reduction manoeuvres. On TSS regions with higher than usual density of traffic and smaller distances between ships, the course alterations alone are not always sufficient or effective means of collision avoidance. Therefore they are supplemented by speed reduction manoeuvres, when necessary. The paper covers all aspects of incorporating speed reduction manoeuvres into the evolutionary method, including generating the initial population, the evaluation phase and the general scheme of the evolutionary cycle.

\section{OPTIMISATION PROBLEM}

The goal of the optimisation process is to find a set of trajectories, which minimizes the average time loss or way loss spent on manoeuvring, while fulfilling the following conditions:
- none of the stationary constraints (including TSS Inshore Traffic Zone [ITZ] and separation zones) are violated, none of the ship domains are violated [4],

- minimum and maximum alteration values are configurable and by default are set to 15 and 60 degrees respectively,

- a ship only manoeuvres when she is obliged to and, in case of head-on and crossing encounters, manoeuvres to starboard are favoured over manoeuvres to port,

- COLREGS rules [3, 5] are not violated (especially Rule 10 and Rules 13 to 17 ),

- speed alterations are not to be applied unless necessary (collision cannot be avoided by a configured maximum course alteration value),

- if speed alteration has to be applied, the number of speed alterations should be minimized (e.g. a ship can reduce her speed to avoid collision and get back to a normal speed once the situation is safe again).

It is assumed that we are given the following data:

- stationary constraints (landmasses and other obstacles and the locations and parameters of each TSS's parts),

- positions, courses and speeds of all ships involved,

- additional ship parameters used for estimating the manoeuvre's dynamics and ship domains

The method directly handles the following COLREGS [5] rules: Rule 13 (Overtaking), Rule 14 (Head-on Situations), Rule 15 (Crossing Situations), Rule 16 (The Give-Way Vessel) and Rule 17(The Stand-On Vessel). Apart from them, the updated version of the method presented here also applies Rule 10 of COLREGS, which governs the behaviour of ships within a TSS [1]. 


\section{WHEN IS SPEED REDUCTION NECESSARY?}

In this section some example scenarios are shown, where it is not possible or not economic to avoid collision by course alteration alone. In general, such situations may be divided into four cases:

- course alterations alone are not economic - large manoeuvres are necessary,

- course alterations alone are misleading - multiple or strange looking manoeuvres are necessary, which might not be understandable for other ships,

- course alterations alone are not sufficient because it is too late - the ships are already too close to each other or their speeds are too large,

- course alterations alone are not possible because there is not enough room to perform them (due to stationary constraints such as safety isobaths or width of traffic lanes).

Examples of situations when course alterations alone are not sufficient are presented below. All scenarios are set in the Gulf of Gdansk TSS (fully shown in Figure 13). For all scenarios the ship domains have been set to values which enable two ships only to transit through a lane parallel to each other.

Scenario 1. positions, courses and speeds are given in Fig. 1. A ship, which is about to cross traffic lanes encounters two ships, which are about to use the incoming lane.

The result (the final set of trajectories) returned by the method, which does not apply speed alterations, is shown in Fig. 2. To avoid collision the crossing ship partially uses the outgoing lane and avoids violating the incoming lane, but its trajectory is considerably longer than would be in case of speed reduction and the manoeuvres of the crossing ship might be misleading for other ships

Scenario 2. Ships positions, courses and speeds are given in Fig. 3. A faster ship encounters two slower ships, one on its starboard, the other on its port board. The result (the final set of trajectories) returned by the method, which does not apply speed alterations, is shown in Fig. 4. The faster ship cannot overtake the slower ships on the incoming lane due to its limited

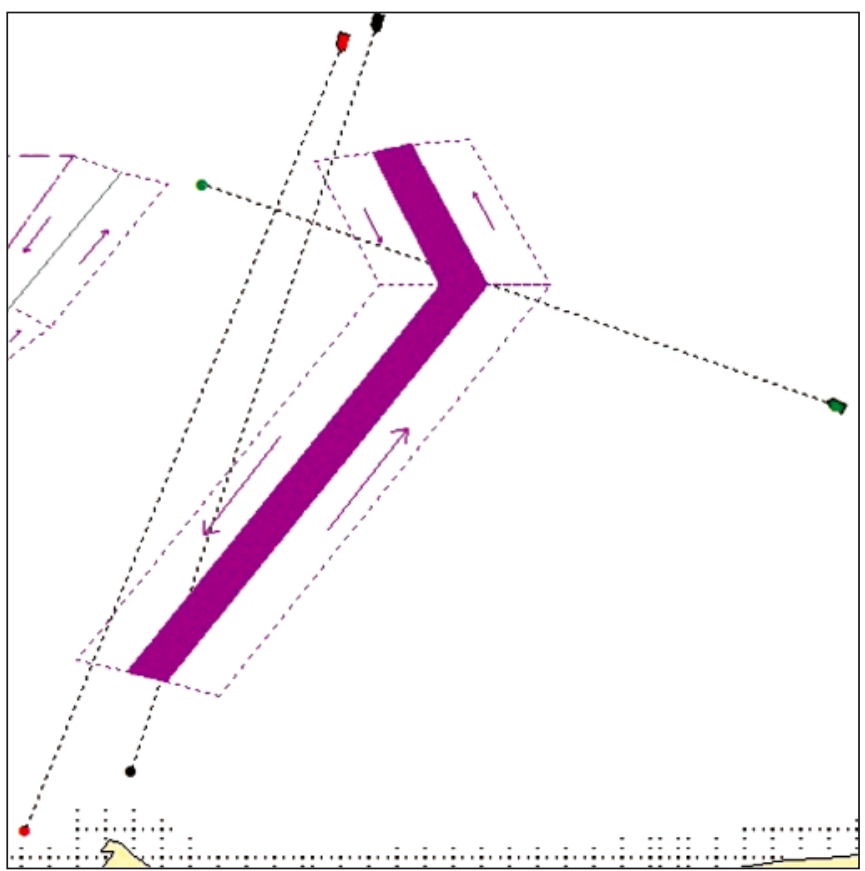

Fig. 1. Scenario 1: Three ships in an encounter situation, speeds (left to right): 14, 14 and 14 knots width, so performs the overtaking manoeuvre outside the lane and joins the lane at later stage.

Scenario 3. Ships positions, courses and speeds are given in Fig. 5. This time a faster ship encounters three slower ships which makes it impossible to use the closest outgoing lane. The result (the final set of trajectories) returned by the method, which does not apply speed alterations, is shown in Fig. 6. The faster ship performs a wide course alteration manoeuvre to starboard so as to avoid collision with the slower ships and avoid violating the incoming lane and the separation zone. In case of enlarging the lane encouragement factor - formula (4) to enforce using the outgoing lane, the method returns alternative solution (Fig. 7), a distant outgoing lane is used and the way loss is significant.

\section{TRAJECTORY PLANNING WITH SPEED REDUCTION}

The possible approaches to applying the speed reduction in the evolutionary method, which have been considered, are as follows:

1. Allowing ships for speed alterations throughout the encounter situation. This means adding another dimension (speed) to the search space, which originally had two dimensions for each node (its geographical coordinates). Conceptually it is the simplest and most natural approach because speed alteration manoeuvres are planned automatically by evolutionary algorithm. Unfortunately it has the following disadvantages:

- may lead to numerous speed alterations thus violating COLREGS which allows for speed alteration only when necessary,

- assumes than any speed within a given range is possible, which might not always be true (sometimes discreet speed values may only be possible depending on the propeller's type),

- largely increases the computational time because of this additional dimension of the computational space.

2. Allowing for one speed reduction manoeuvre only, chosen from a predefined set, and then getting back to the

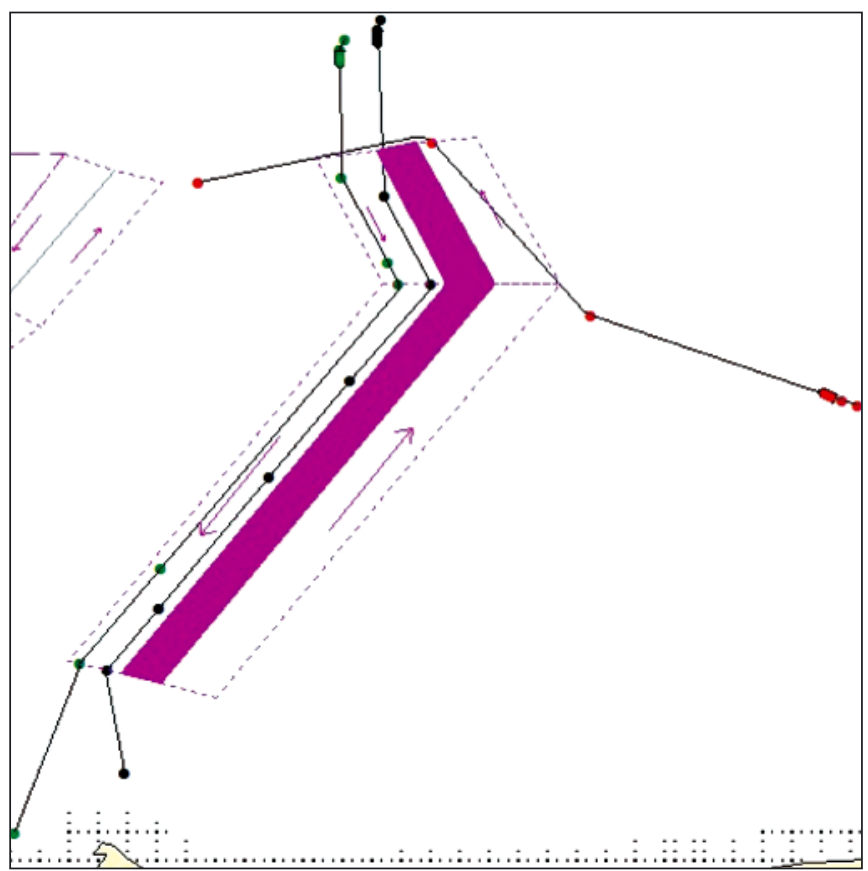

Fig. 2. A solution returned by the method without speed reduction for Scenario 2 


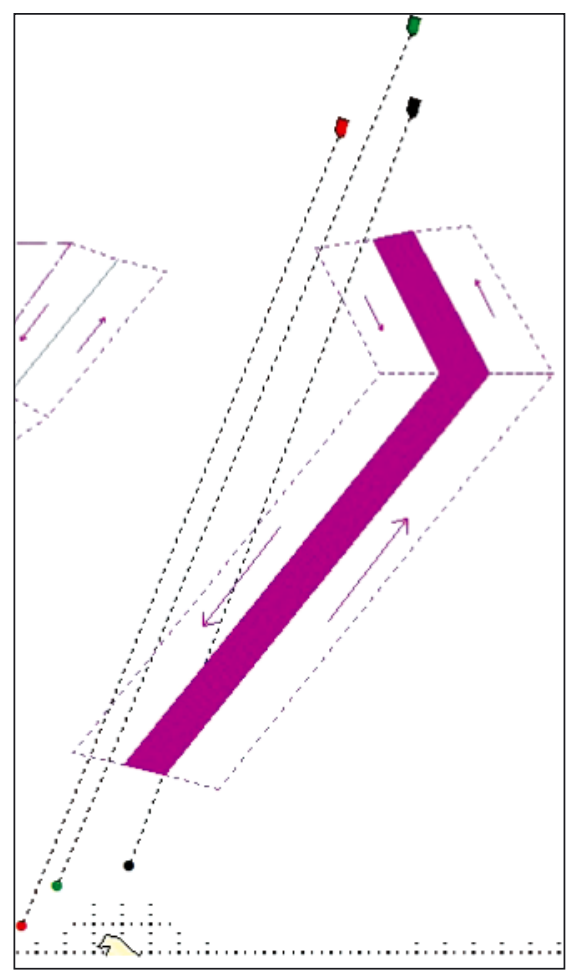

Fig. 3. Scenario 2: Three ships in an encounter situation, speeds (left to right): 10, 15 and 10 knots

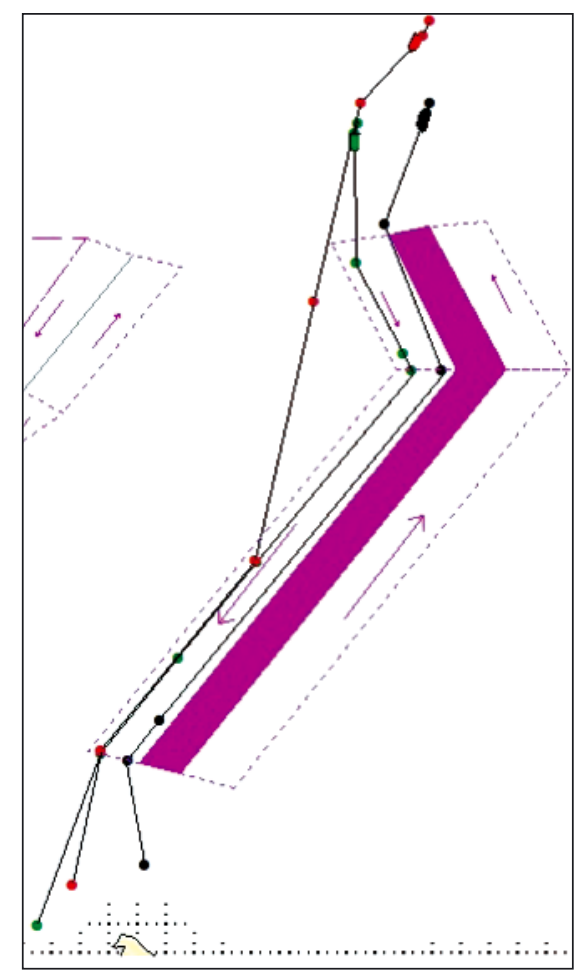

Fig. 4. A solution returned by the method without speed reduction for Scenario 2

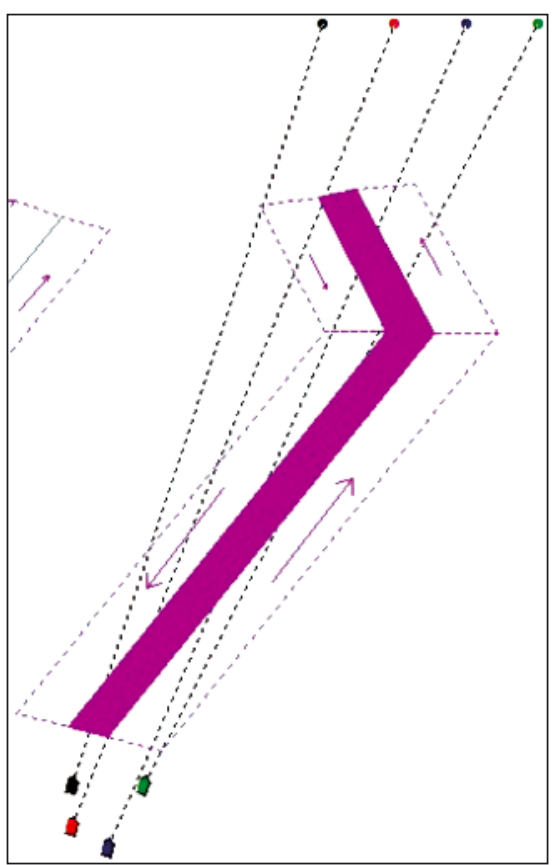

Fig. 5. Scenario 3: Four ships in an encounter situation, speeds (left to right): 10, 12, 10 and 10 knots

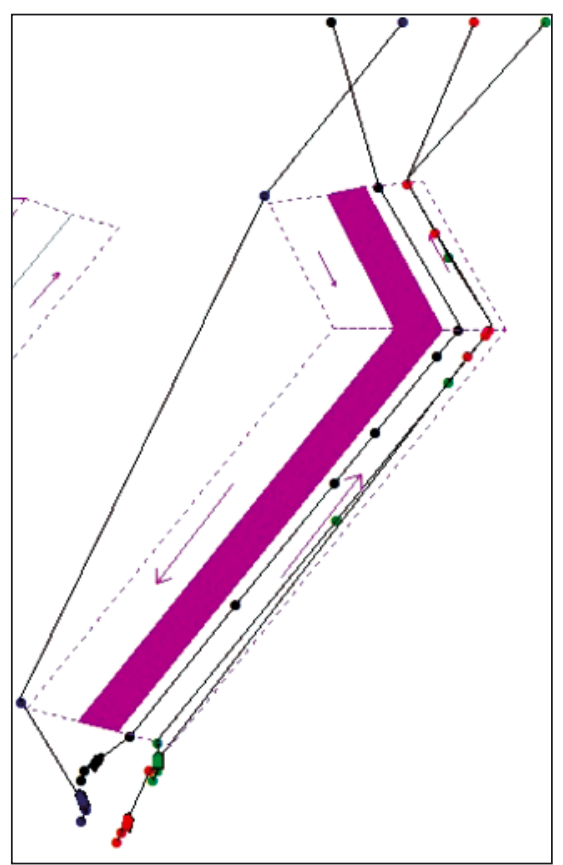

Fig. 6. A solution returned by the method without speed reduction for Scenario 3

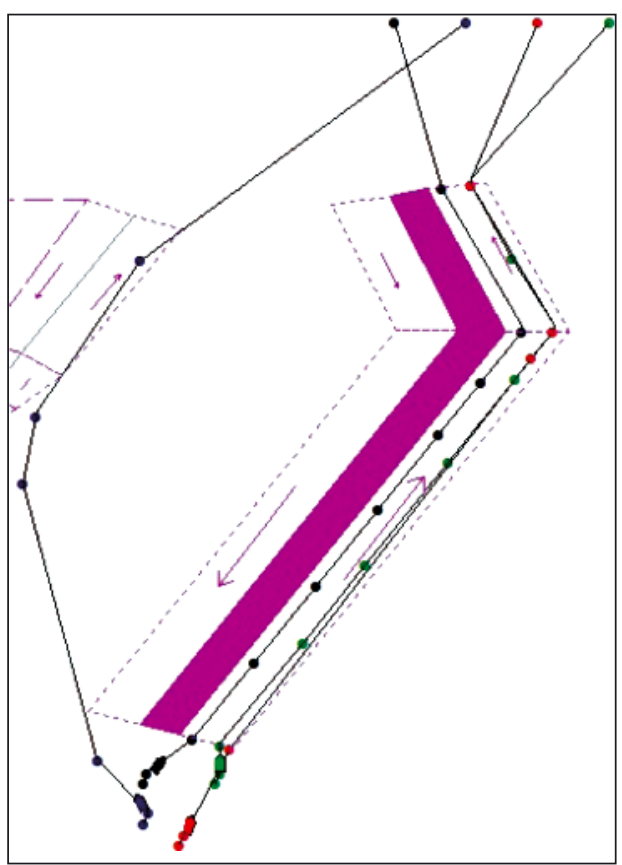

Fig. 7. An alternative solution returned by the method without speed reduction for Scenario 3 original speed when possible. This approach is void of the disadvantages typical for the previous one:

- it naturally supports discreet speed values,

- it keeps the constant number of speed alterations equal 2

- it keeps reasonable computational space and time.

Unfortunately this approach involves applying speed reduction manoeuvres partially outside of the main evolutionary algorithm, which limits the benefits from the evolutionary process.
Taking into account all abovementioned factors, the author has decided to follow the second approach. It is described more thoroughly below.

\section{A new scheme of the ESoSST method}

The basic scheme of the ESoSST method without speed reduction is shown in Fig. 8. As can be seen, Specialised operators and mutation is preceding reproduction here, as opposed to the traditional approach. The reason for this is that specialised operators utilize information from evaluation and 
therefore they cannot be applied after reproduction (which introduces new individuals into population) but can be applied after succession (which only reduces the number of individuals without affecting any of them).

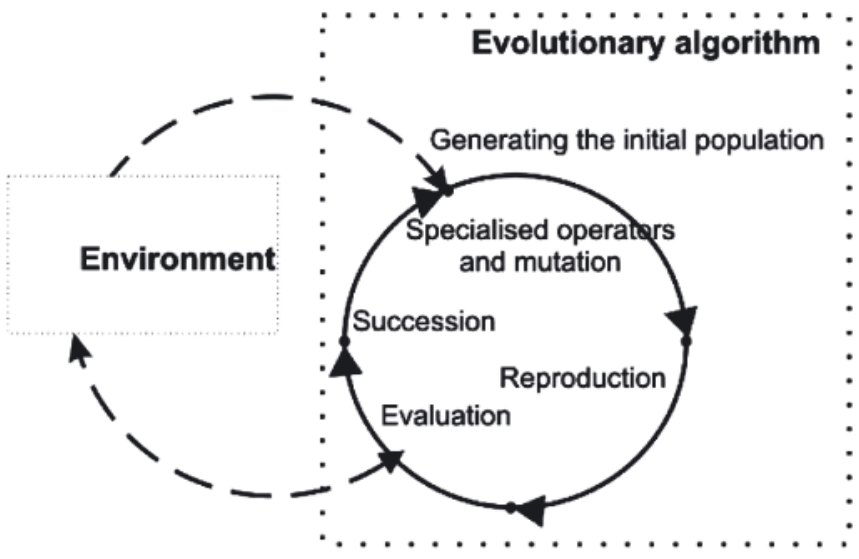

Fig. 8. The scheme of EA used by ESoSST method without speed reduction

To apply speed reduction this scheme is extended by additional operations shown in Fig. 9.

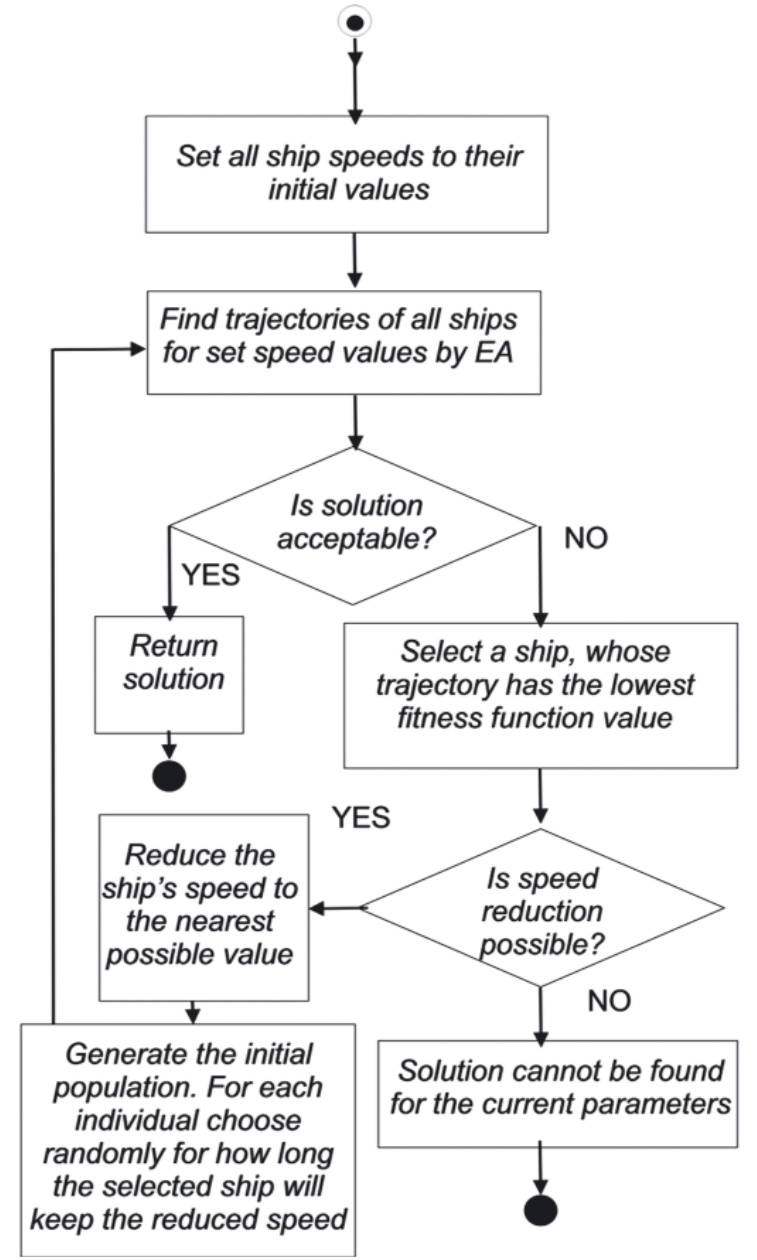

Fig. 9. The ESoSST method with speed reduction

This new algorithm works as follows:

1. The EA is run normally as depicted in Fig. 8.

2. If the results are unsatisfactory and indicate that using course alterations alone does not suffice, then EA is run again but with the following changes.

a. The previously found highest-rated set of ship trajectories is analysed and the speed of the ship which had lowest-rated trajectory (when compared to other ships in the set) will have its speed reduced (changed to the nearest possible speed, e.g. $70 \%$ of the original speed).

b. The reduced speed is to be applied after the preconfigured decision time and it is kept for a randomly chosen number of segments. This is a part of the process of generating the initial population. As a result, each of the initial sets of ship trajectories has the selected ship travelling at the reduced speed for a number of segments and then getting back to the original speed again.

c. When evaluating the sets of ship trajectories, the new value of the selected ship's speeds is considered, when necessary to determine approach factors for potential collisions with other ships. The new values of the selected ship's speed affects the total passage time, which is also taken into account when computing the fitness function value (1).

3. If the results are still unsatisfactory and still indicate that using course alterations alone might be the reason of the problem, then EA is run once more.

a. The previously found highest-rated set of ship trajectories is analysed again. If the ship having the lowest-fitted trajectory is the same as before, than the procedure described in points $2 \mathrm{a}$ to $2 \mathrm{c}$. is repeated for the further reduced speed (e.g. $50 \%$ of the original value).

4. If the results are still unsatisfactory and the ship having the lowest-fitted trajectory cannot have its speed reduced any further then the method returns the best of the previously found sets of trajectories accompanied with a message that acceptable solution could not been found.

\section{Fitness function of the ESoSST method}

The formula for the fitness function including speed reduction manoeuvres is as follows.

$$
f=\sum_{i=1}^{n}\left[f_{i}\right]
$$

where:

$$
\mathrm{f}_{\mathrm{i}}=\mathrm{e}_{\mathrm{i}} \mathrm{s}_{\mathrm{i}} \mathrm{a}_{\mathrm{i}} \mathrm{c}_{\mathrm{i}} \mathrm{t}_{\mathrm{i}}
$$

$\mathrm{s}_{\mathrm{i}}$ (static constraint factor), $\mathrm{a}_{\mathrm{i}}$ (collision avoidance factor), $\mathrm{c}_{\mathrm{i}}$ (COLREGS-compliance factor) have already been described in [10]. Symbol $\mathrm{e}_{\mathrm{i}}$ (trajectory economy factor) is computed in a different way for ships which have to reduce their speed than for those, which do not.

For changing propeller's settings, that is for ships, which reduce their speed:

$$
e_{i}=\left(\frac{l_{i}-w_{i}}{l_{i}}\right)
$$

where:

$\mathrm{i}$ - the index of the current ship,

$1_{i}-$ the total length of the i-th ship's trajectory [nautical miles].

$\mathrm{w}_{\mathrm{i}}$ - the difference between the length of the i-th ship's trajectory and the length of a predetermined trajectory of the i-th ship (obtained without other ships affecting $\mathrm{j}$-th ship trajectory) [nautical miles].

For fixed propeller's settings, that is for ships which do not reduce their speed time and time loss are used instead of length and way loss respectively. In case of speed reduction, the trajectory economy factor would be much lower when 
using total time and time loss. This would lead to unacceptably low trajectory fitness value and total fitness function value. Consequently, the algorithm (Fig. 10) would make the same ship further reduce its speed, even when not necessary. To avoid it, the length-oriented trajectory economy factor (3) is used for ships, which reduce their speed.

Symbol $t_{\mathrm{i}}$ factor from formula (2) is responsible for TSScompliance and is computed as given by (4).

where:

$$
\mathrm{t}_{\mathrm{i}}=\left(1-\sum_{\mathrm{k}=1}^{\mathrm{m}}\left[\mathrm{p}_{\mathrm{k}}\right]\right) *\left[1+\mathrm{r}_{\mathrm{i}}(\mathrm{g}-1)\right]
$$

$\mathrm{m}$ - the number of TSS rules violations registered for the current ship,

$\mathrm{k}$ - the index of a registered violation,

$\mathrm{p}_{\mathrm{k}}$ - the penalty for the $\mathrm{k}$-th of the registered TSS rules violations,

$\mathrm{g}$ - lane encouragement factor applied to encourage using traffic lanes, set to 1.5 by default,

$r_{i}$ - trajectory's lane percentage factor (a percentage of the trajectory's length that transits through a traffic lane).

\section{Ship priority groups}

An additional extension to the ESoSST scheme is introducing ship priority groups. They are used for convenience of the system's user as well as for optimizing the method's performance. The number of ships within a TSS or within a region supervised by a VTS centre can be quite large and finding an optimal set of all trajectories within a single algorithm run would be time consuming. Instead, depending on the ships' parameters and the areas of a TSS, which they transit, ships can be divided into priority groups to save on computational time (two or three smaller groups of ships will be processed faster than one large group). In the ESoSST method a two-digit priority numbering is used, where the first digit denotes the class of service for a given ship and the second digit - the priority number within this class. Ships with dangerous loads or limited manoeuvring capability would be privileged by being assigned a lower first digit and their trajectories would be planned first. Other ships trajectories would then be planned in such a way so as not to collide with each other and with the trajectories planned previously. The second digit might be assigned manually or automatically (based on the area of a TSS or traffic lane, which the ship is supposed to transit through). An example is presented in Fig. 10.

In Fig. 13 three ships are shown. Two of them will traverse the west (left) part of the TSS and one will traverse the east (right) part of the TSS. It is assumed that the first of the ships traversing the west part of the TSS is carrying a dangerous load and therefore is assigned a higher priority (lower first digit). As a result the ships are given priority numbers: 11,21 and 22 and their routes would be planned in the ascending order of these numbers.

When ship priority groups are applied, the operations presented previously on the algorithm from Fig. 10 are performed in turns for each of the priority groups. The trajectories of ships from each priority groups are checked for potential collisions with each other as well as with the trajectories already found for previous priority groups.

\section{EVOLUTIONARY OPERATORS AND TECHNIQUES}

In this section details on elements of the evolutionary method from Fig. 11 are described. The evaluation (fitness function) has already been presented, so the remaining elements are: generating the initial population, specialised operators and mutation, reproduction and selection.

\section{An individual's structure and generating the initial population}

Each individual consists of a set of trajectories, each of them representing one of the ships involved in an encounter situation. Each trajectory is a sequence of nodes containing geographical coordinates $\mathrm{X}$ and $\mathrm{Y}$.

Apart from straight segments and randomly generated individuals used in previous versions of the ESoSSt method, in the TSS-oriented version the initial population also includes

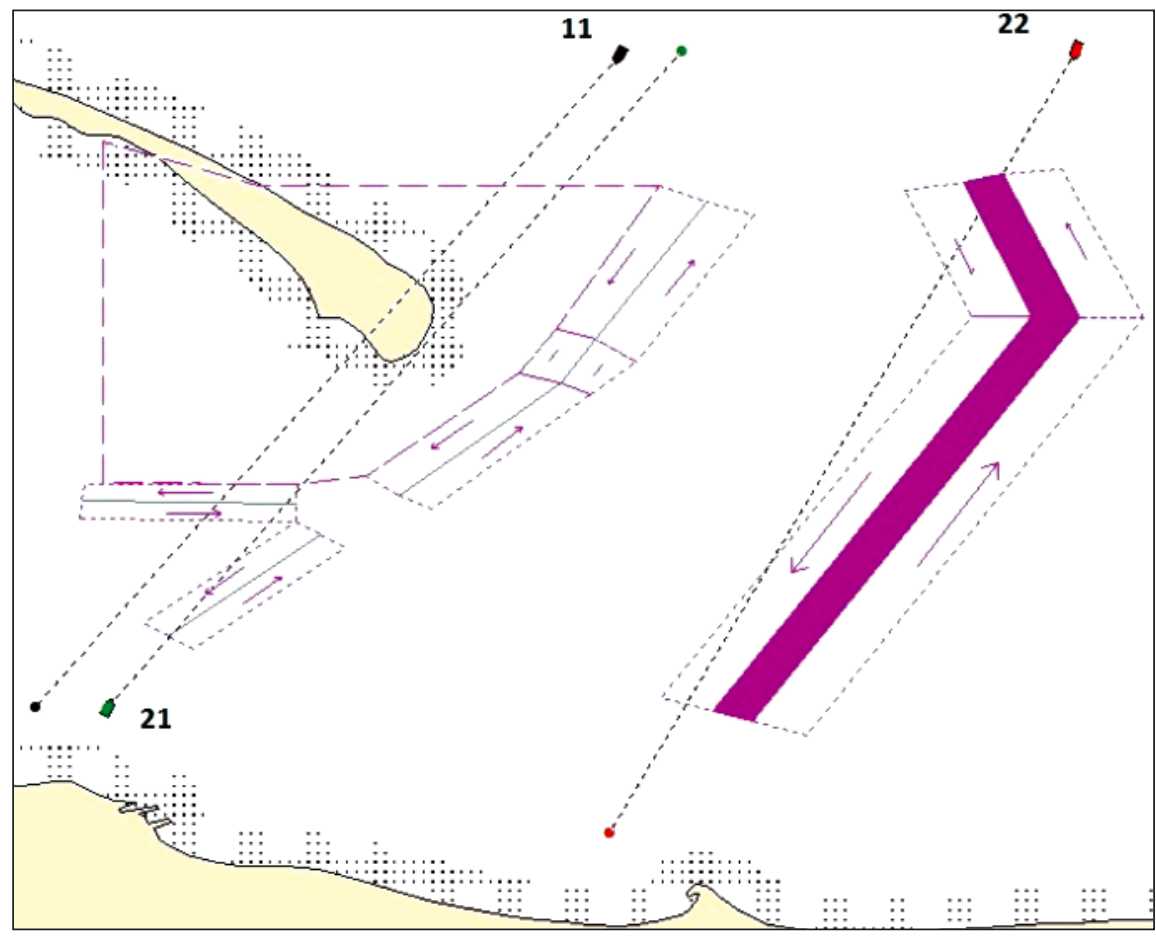

Fig. 10. Three ships and the priority numbers assigned to them by the method 
individuals consisting of predefined, TSS-compliant tracks, generated automatically for each ship. The random sets of trajectories, which constitute the majority of the initial population, are necessary for wide exploration, while the predefined tracks result in a faster convergence to a solution.

The size of the population is configurable (100 by default). It doubles during specialised operators and mutation phase and then doubles again during reproduction. It is reduced to its original size during succession.

\section{Specialised operators and mutation}

Four types of mutation operators (node insertion, nodes joining, node shift and node deletion) have been used. More important than them however are the specialised operators designed to handle particular problems and selected depending on the particular situation (time remaining to collision etc.). They include five types of ship collision-avoidance operators (segment insertion, segment shift, first node shift, second node shift and node insertion) all of which are parameterised and semi-deterministic as opposed to random mutation operators. Analogically to ship collision avoidance operators, five kinds of operators avoiding collisions with static obstacles have been used. Apart from that, three types of additional operators (reduction of unnecessary nodes, smoothing and adjusting) have been applied. The TSS-oriented version of the method includes ten new operators handling TSS-violations. Altogether, twenty seven different operators have been used, the majority of them being problem-dedicated. Problemdedicated operators were applied always if collision or rule violation was registered. Only in case of a lack of need for applying a specialised operator, mutation operators were used. The probability of mutation decreased with the increase of fitness function value of a particular trajectory of an individual, so as to improve low-fitted trajectories without spoiling the high fitted ones.

\section{Reproduction}

In the reproduction phase pairs of individuals (parents) are crossed to generate new individuals (offspring). Four types of crossover operators have been designed and implemented:

a) An offspring inherits whole trajectories from both parents and the higher-valued of the two possible trajectories is chosen.

b) An offspring inherits whole trajectories from both parents and the choice of a particular trajectory (from the first or the second parent) is done randomly.

c) Each of the trajectories of the offspring is a crossover of the appropriate trajectories of the parents.

d) Each node of a trajectory is an arithmetical crossover of the nodes in the parents' trajectories.

Of these four types the first one has been eliminated eventually because it involved doubling the evaluation phase and its benefits were not enough to justify the this additional computational cost.

\section{Selection}

Prior to implementing the TSS-oriented version of the method various methods of pre-selection (selection for crossover purpose) and post-selection (selection for succession) purpose have been tested including proportional (roulette wheel) and modified proportional (modified roulette wheel). Eventually uniform pre-selection and threshold post-selection were chosen as their results were competitive and they worked were faster than proportional and modified proportional.

\section{Number of generations and the computational time}

The process was stopped if a satisfying (configurable) value of a normalized fitness function was reached ( 0.99 by default) and there were no collisions or rule violations registered. Otherwise, the stop criterion of a maximum number of generations or maximum time was used. In case of a collision threat, an Officer Of the Watch (OOW) should make a decision and initiate a manoeuvre within 6 minutes. Therefore a 1-minute time for returning the results was chosen: it should satisfy the needs of navigators and VTS operators alike. In practice, it allowed for 100-200 generations (depending on the number of ships) and the process would nearly always converge by then and the total fitness function would be above 0.97 (way loss below 3\%). This was due to the specialised operators and predefined tracks which greatly increased the convergence rate, though occasionally at the cost of a slightly larger way loss (up to 5\%). All experiments were carried out on a standard PC machine (processor Intel Core i7-2600k, $3.40 \mathrm{GHz}, 8$ GB RAM).

\section{SOLVING THE ENCOUNTER SITUATIONS BY THE ESOSST METHOD WITH SPEED REDUCTION MANOEUVRES}

In this section the examples of situations previously shown as unsolvable by the method without speed reduction, are now dealt with by the extended version of the ESoSST method.

Scenario 1. The result (the final set of trajectories) returned by the method with speed reduction is shown in Fig. 11. The crossing ship reduces its speed and passes safely astern of the two ships, which transit the incoming lane.

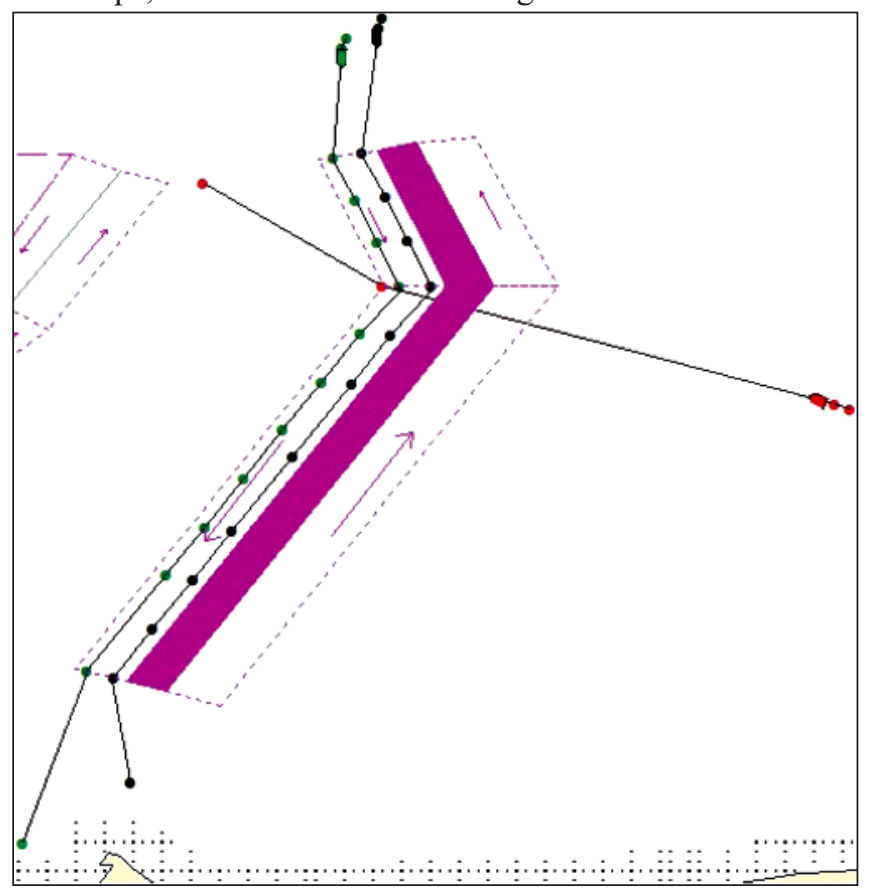

Fig. 11. A solution returned by the method with speed reduction for Scenario 1

Scenario 2. The result (the final set of trajectories) returned by the method with speed reduction is shown in Fig. 12. The faster ship reduces its speed which enables it to use the outgoing lane as recommended by COLREGS. 


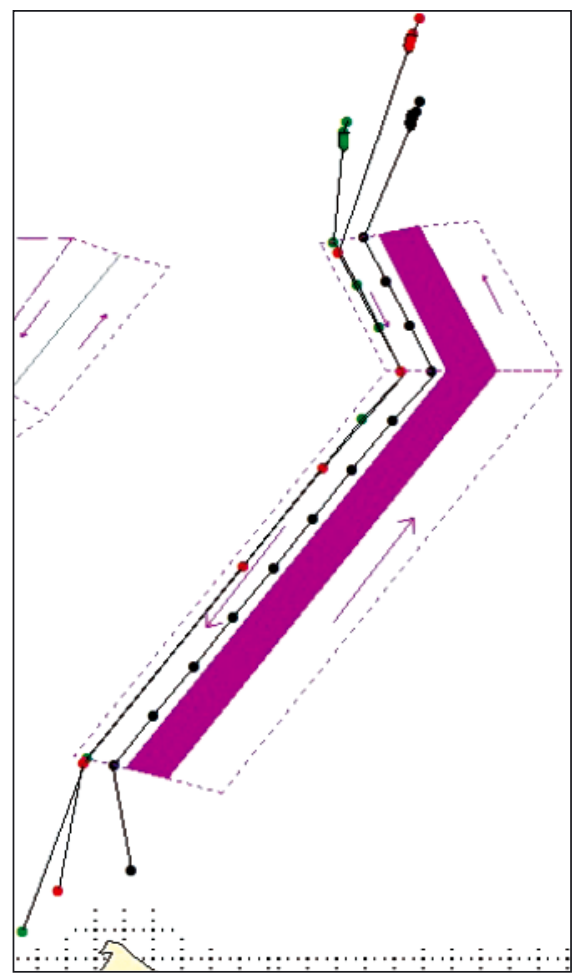

Fig. 12. A solution returned by the method with speed reduction for Scenario 2

Scenario 3. The result (the final set of trajectories) returned by the method with speed reduction is shown in Fig. 13. Similarly to Scenario 2, the faster ship reduces its speed which enables it to use the outgoing lane as recommended by COLREGS.

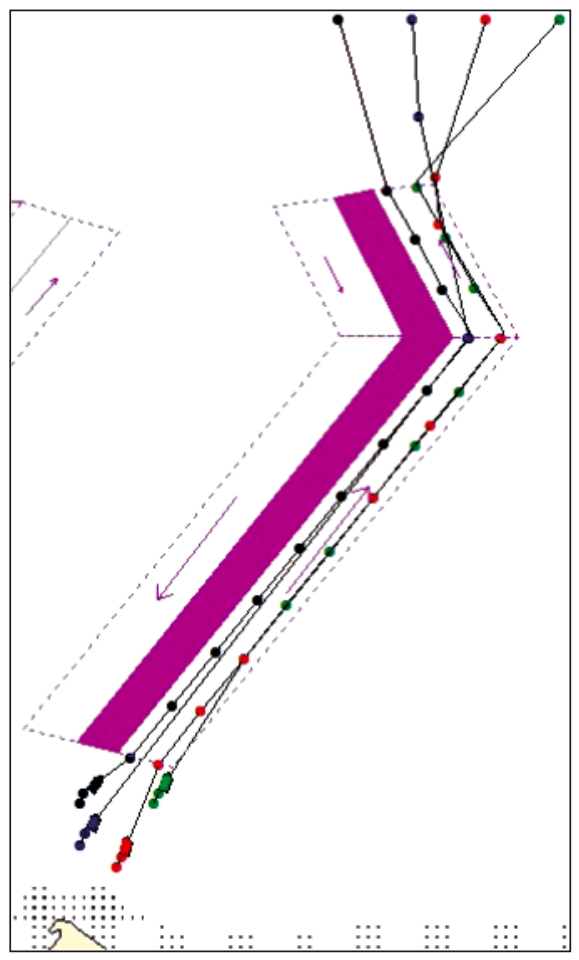

Fig. 13. A solution returned by the method with speed reduction for Scenario 3

In general in all of the test scenarios speed reduction enabled larger number of ships to use traffic lanes in a way recommended by COLREGS, either transiting the lanes or crossing them while avoiding collisions. The way loss spent on collision avoidance maneuvers was insignificant (always below $3 \%$ ) and therefore all results can be considered satisfactory. It was additionally tested that further evolution (larger number of generations) would only bring insignificant rise in fitness value (below $0.5 \%$ ) or no rise at all.

\section{SUMMARY AND CONCLUSIONS}

In the paper an extended version of the author's ESoSST method has been presented. The method works for Traffic Separation Scheme and applies speed reduction manoeuvres when necessary. The experiments that have been carried out confirmed the correctness of the presented approach to speed reduction problem:

- speed reduction manoeuvres are only applied when necessary,

- applying speed reduction effectively solves encounter situation in situations when course alterations alone are not sufficient or would result in significant way loss,

- the presented extension to the method does not affect any of the advantages of the previous version.

The further research on the method will be focused on:

- scalability of this approach (for larger number of ships and larger maps),

- optimising this approach by testing various evolutionary techniques,

- ensuring the reliability of the method, especially partial solutions in situations when the optimisation problem cannot be fully solved due to very strict safety constraints.

\section{BIBLIOGRAPHY}

1. Anwar N., Khalique A.: Passage Planning Principles. Witherby Seamanship International, Livingston, United Kingdom, 2006.

2. Cheng X. and Liu Z.: Trajectory Optimization for Ship Navigation Safety Using Genetic Annealing Algorithm. Proceedings of ICNC 2007 Third International Conference on Natural Computation, 4., 385 - 392, 2007.

3. Cockcroft A.N. and Lameijer J.N.F.: A Guide to Collision Avoidance Rules. Butterworth-Heinemann, 2011.

4. Coldwell T.G.: Marine Traffic Behaviour in Restricted Waters. The Journal of Navigation. 36, 431-444, 1983.

5. COLREGS. Convention on the International Regulations for Preventing Collisions at Sea. International Maritime Organization, London, 1972 (with amendments adopted from December 2009).

6. Ito M., Feifei Z., Yoshida N.: Collision avoidance control of ship with genetic algorithm. Proceedings of the 1999 IEEE International Conference on Control Applications, vol. 2, 17911796, 1999.

7. Lisowski J.: The Dynamic Game Models of Safe Navigation, International Journal on Marine Navigation and Safety of Sea Transportation, 1, no. 1., 11-18, 2007.

8. Statheros T., Howells G. and McDonald-Maier K.: Autonomous Ship Collision Avoidance Navigation Concepts, Technologies and Techniques, The Journal of Navigation, 61, 129-142, 2008.

9. Smierzchalski R., Michalewicz Z.: Modelling of a Ship Trajectory in Collision Situations at Sea by Evolutionary Algorithm, IEEE Transactions on Evolutionary Computation. No. 3 Vol. 4, 227-241, 2000.

10.Szlapczynski R.: Evolutionary Sets of Safe Ship Trajectories within Traffic Separation Schemes. The Journal of Navigation. vol. 66, iss. 1 65-81, 2012.

11.Tam C.K. and Bucknall R.: Path-Planning Algorithm for Ships in Close-Range Encounters. Journal of Marine Science Technology. 15, 395-407, 2010. 
12.Tsou M. C. and Hsueh C. K.: The Study of Ship Collision Avoidance Route Planning by Ant Colony Algorithm. Journal of Marine Science and Technology. 18(5), 746-756, 2010a.

13.Tsou M. C., Kao S.L. and Su C.M.: Decision Support from Genetic Algorithms for Ship Collision Avoidance Route Planning and Alerts. The Journal of Navigation, 63, 167-182, 2010 b.

14.Xue Y., Lee B.S. and Han D.: Automatic Collision Avoidance of Ships. Proceedings of the Institution of Mechanical Engineers, Part M: Journal of Engineering for the Maritime Environment, 33-46, 2009.

15.Yang L. L., Cao S. H. and Li B.Z.: A Summary of Studies on the Automation of Ship Collision Avoidance Intelligence. Journal of Jimei University (Natural Science), 2006.
16.Zeng X.: Evolution of the Safe Path for Ship Navigation. Applied Artificial Intelligence. 17., 87-104, 2003.

\section{CONTACT WITH AUTHOR}

Rafał Szłapczyński, Ph. D. Faculty of Ocean Engineering and Ship Technology,

Gdańsk University of Technology Narutowicza 11/12

80-233 Gdańsk, POLAND e-mail: rafal@pg.gda.pl 